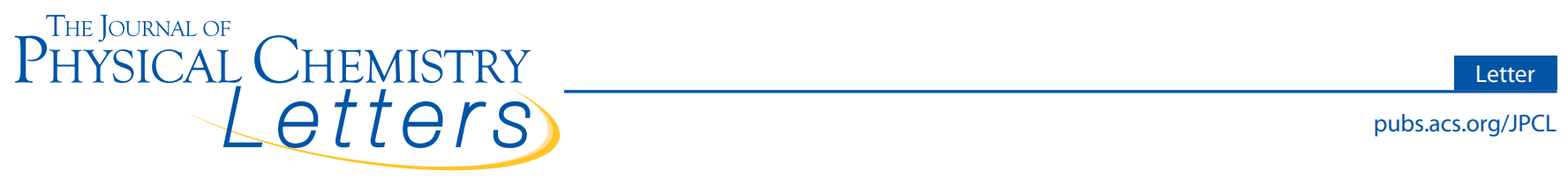

\title{
New Phases of Germanene
}

\author{
V. Ongun Özçelik, ${ }^{\dagger, \ddagger}$ E. Durgun, ${ }^{\dagger \neq}$ and Salim Ciraci*, ${ }^{\mathbb{I}}$

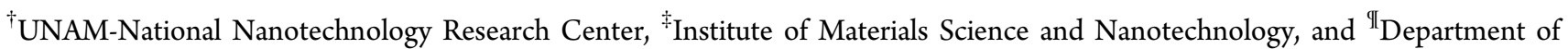 \\ Physics, Bilkent University, 06800 Ankara, Turkey
}

Supporting Information

ABSTRACT: Germanene, a graphene-like single-layer structure of $\mathrm{Ge}$, has been shown to be stable and recently grown on $\mathrm{Pt}$ and $\mathrm{Au}$ substrates. We show that a $\mathrm{Ge}$ adatom adsorbed on germanene pushes down the host $\mathrm{Ge}$ atom underneath and forms a dumbbell structure. This exothermic process occurs spontaneously. The attractive dumbbell-dumbbell interaction favors high coverage of dumbbells. This Letter heralds stable new phases of germanene, which are constructed from periodically repeating coverage of dumbbell structures and display diversity of electronic and magnetic properties.

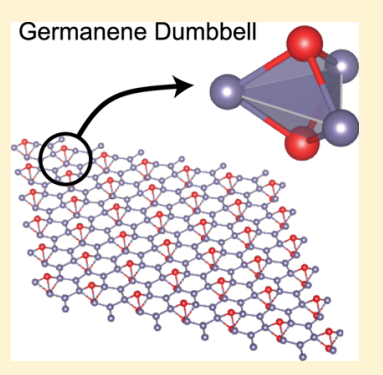

SECTION: Physical Processes in Nanomaterials and Nanostructures

$\mathrm{T}$

hree-dimensional (3D) layered bulk phases, such as graphite, ${ }^{1} \mathrm{BN},{ }^{2}$ and $\mathrm{MoS}_{2},{ }^{3}$ have led to the synthesis of single-layer, honeycomb structures of those materials, which were initially conjectured to be unstable. ${ }^{4-7}$ The existence of single-layer, graphene-like structures of other group IV elements, like $\mathrm{Si}$ and $\mathrm{Ge}$, have been ruled out because these elements do not have layered allotropes like graphite that would allow the synthesis of their single-layer structures. Surprisingly, on the basis of state of the art first-principles calculations, silicene, ${ }^{8,9}$ germanene, ${ }^{10,11}$ most of the III-V and II-VI compounds, ${ }^{9,11}$ and several transition-metal dichalcogenides and oxides ${ }^{12}$ have been shown to form stable, single-layer honeycomb structures. Moreover, it has been also shown that silicene and germanene share several of the exceptional properties of graphene, such as $\pi$ and $\pi^{*}$ bands linearly crossing at the Fermi level and hence forming Dirac cones, the ambipolar effect, and the family behavior observed in nanoribbons. ${ }^{10,13}$ Advances in growth techniques have enabled the synthesis of some of these predicted single-layer structures, in particular, the growth of single- and multilayer silicene on $\mathrm{Ag}(111)$ substrates ${ }^{14-16}$ and the growth of germanene on $\mathrm{Pt}$ and $\mathrm{Au}$ substrates ${ }^{17-19}$ were recently succeeded.

In this Letter, we find that a single Ge adatom adsorbed to germanene constructs a dumbbell (DB) structure. Even more remarkable is that new phases can be constructed by the periodic coverage of germanene with DBs. Depending on the coverage of DBs, these stable phases can be metal or narrow band gap semiconductors in magnetic or nonmagnetic states, and hence, they attribute diverse functionalities to germanene. We believe that the intriguing stacking of these phases can pave the way to the layered phase of bulk germanium, that is, germanite.

Our predictions are obtained from the state of the art firstprinciples pseudopotential calculations based on the spinpolarized density functional theory (DFT) within the generalized gradient approximation (GGA) including van der
Waals corrections. ${ }^{20}$ We used projector-augmented wave potentials (PAWs), ${ }^{21}$ and the exchange-correlation potential is approximated with the Perdew-Burke-Ernzerhof (PBE) functional. $^{22}$ The Brillouin zone (BZ) was sampled in the Monkhorst-Pack scheme, where the convergence in energy as a function of the number of $k$-points was tested. The $k$-point sampling of $(21 \times 21 \times 1)$ was found to be suitable for the BZ corresponding to the primitive unit cell of germanene. For larger supercells, this sampling has been scaled accordingly. The number of $k$-points was further increased to $(48 \times 48 \times 1)$ in the density of states calculation. Atomic positions were optimized using the conjugate gradient method, where the total energy and atomic forces were minimized. The energy convergence value between two consecutive steps was chosen as $10^{-5} \mathrm{eV}$. A maximum force of $0.002 \mathrm{eV} / \AA$ was allowed on each atom. Numerical calculations were carried out using the VASP software. ${ }^{23}$ Because the band gaps are underestimated by DFT, we also carried out calculations using the HSE06 hybrid functional, ${ }^{24}$ which is constructed by mixing $25 \%$ of the Fock exchange with $75 \%$ of the PBE exchange and $100 \%$ of the PBE correlation energy. The phonon dispersion curves were calculated using the small displacement method. ${ }^{25}$

It is known that the splitting of the three-fold valence band at the center of the BZ due to SOC is significant $(290 \mathrm{meV})$ for the Ge crystal in a cubic diamond (cdGe) structure. ${ }^{26}$ The spin-orbit splitting in germanene is crucial as a starting point of the present study. Despite $1 \mathrm{meV}$ spin-orbit splitting in silicene, ${ }^{27}$ the spin-orbit splitting of germanene at $\Gamma$ - and $\mathrm{K}$ points of the hexagonal $\mathrm{BZ}$ are calculated to be 70 and $23 \mathrm{meV}$. Accordingly, it is set that at the $\mathrm{K}, \mathrm{K}^{\prime}$-points, where the linear bands cross at the Fermi level in the absence of SOC, a gap of

Received: May 16, 2014

Accepted: July 11, 2014

Published: July 11, 2014 
$23 \mathrm{meV}$ is opened. This way, the semimetallic state and massless Fermion behavior of electrons of pristine germanene are discarded, as seen in Figure 1. This result is critical for our study dealing with new derivatives of pristine germanene.

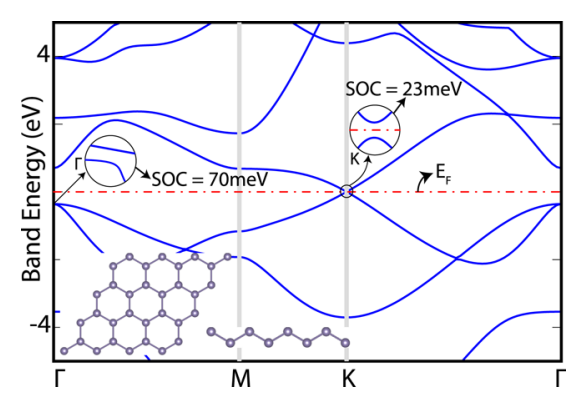

Figure 1. Electronic structure of monolayer germanene. The splittings due to the spin-orbit coupling (SOC) are shown in the magnified insets. The Fermi level is set to the zero of energy and is indicated by the dashed-dotted line. The atomic structure of buckled germanene is illustrated by the inset.

Having clarified the effect of SOC on pristine germanene, we next examine the construction of a single DB structure. In Figure 2a, we present various stages of conjugate gradient


Figure 2. (a) Snapshots of conjugate gradient steps in the course of the formation of a DB structure. The external Ge adatom first approaches to the germanene layer from the top site and eventually constructs the DB structure by pushing the host Ge atom down. (b) Top and side views of the DB formed on $(4 \times 4)$ germanene. Two Ge atoms of the DB are highlighted by red. (c) The DB zoomed in along with the total charge density isosurfaces. (d) Contour plots of the total charge density on planes passing through $\mathrm{D}_{1}-\mathrm{A}-\mathrm{D}_{2}$ and $\mathrm{B}-\mathrm{D}_{2}-\mathrm{C}$ atoms. Note that although the DB atoms make bonds with nearest germanene atoms, there is no bonding between the $\mathrm{DB}$ atoms $\mathrm{D}_{1}$ and $\mathrm{D}_{2}$.

calculations taking place in the course of the adsorption of a single Ge adatom. In the presence of an external and free $\mathrm{Ge}$ adatom, the formation of a DB structure on germanene is spontaneous. The external Ge adatom eventually moves closer to the germanene surface and makes a bridge bond with two underlying $\mathrm{Ge}$ atoms of germanene. It then starts to push the
Ge atom underneath further down until the final DB forms, as shown in Figure 2a. As a concerted process, two Ge atoms above and below the germanene surface, named $D_{1}$ and $D_{2}$, donate significant electronic charge to the three nearest $\mathrm{Ge}$ atoms of germanene, and hence, each forms three strong $\mathrm{Ge}-$ Ge bonds with a length of $2.58 \AA$ A. With these additional bonds with $\mathrm{DB}$, these three $\mathrm{Ge}$ atoms of germanene become four-foldcoordinated. $\mathrm{D}_{1}$ and $\mathrm{D}_{2}$ by themselves engage in a $3+1$ coordination because each has three nearest-neighbor Ge atoms at a distance of $2.58 \AA$, whereas the $\mathrm{D}_{1}-\mathrm{D}_{2}$ distance of $2.80 \AA$ is slightly larger. At the end, the resulting $\mathrm{DB}$ corresponds to a local minimum on the Born-Oppenheimer surface and remains stable. As for the DB-decorated germanene, it is a structure between the four-fold-coordinated cdGe and the three-fold-coordinated single-layer, buckled germanene. Because moving a DB from one place to another by breaking three $\mathrm{Ge}-\mathrm{Ge}$ bonds involves an energy barrier, any pattern of DBs on germanene is expected to remain stable. It is noted that DB has been found to be the second most energetic defect structure of a carbon adatom on graphene. ${ }^{12}$ Later, it was found to be the most energetic defect structure of a $\mathrm{Si}$ adatom on silicene, ${ }^{28,29}$ and it has been demonstrated that it can form spontaneously without an energy barrier as long as a Si adatom on silicene is available. ${ }^{30}$ In the present Letter, we demonstrate that $\mathrm{Ge} \mathrm{DB}$ can form new phases (or derivatives) of germanene with novel electronic and magnetic properties.

Because the construction of a single DB is an exothermic process and hence does not involve any energy barrier, the formation of a DB structure is unavoidable as long as a free $\mathrm{Ge}$ adatom is present at the close proximity of the surface. We define the associated binding energy as $E_{\mathrm{b}}=E_{\mathrm{T}}$ [germanene $]+$ $E_{\mathrm{T}}[\mathrm{Ge}]-E_{\mathrm{T}}$ [germanene $\left.+\mathrm{DB}\right]$, in terms of the total energies of germanene $+\mathrm{DB}$, pristine germanene, and free Ge adatom. The binding energies are calculated for a single DB in the $(n \times$ $n$ ) hexagonal supercells with varying values of $n$. Accordingly, $E_{\mathrm{b}}$ is the energy gained from the construction of a single DB through the adsorption of a single $\mathrm{Ge}$ adatom to germanene, and $E_{\mathrm{b}}>0$ indicates an exothermic process. For an isolated DB calculated in a large supercell with $n=8, E_{\mathrm{b}} \approx 3.4 \mathrm{eV}$, but it increases with decreasing $n$ or decreasing DB-DB distance due to the attractive interaction among DBs, as discussed in the next paragraph. In Figure $2 b$ and $c$, the atomic configurations of a single $\mathrm{DB}$ and its relevant structural parameters together with isosurfaces of charge density of $\mathrm{Ge}-\mathrm{Ge}$ bonds around $\mathrm{DB}$ are shown. The charge density counter plots calculated on various planes are presented in Figure 2d. The bonding of the DB atoms $\left(D_{1}\right.$ and $\left.D_{2}\right)$ with the nearest $G e$ atoms of germanene are clearly seen. Notably, there is no bonding between $D_{1}$ and $\mathrm{D}_{2}$.

The $\mathrm{DB}-\mathrm{DB}$ interaction on the surface of germanene is crucial for the growth of germanene phases. While an attractive interaction between two DBs can lead to the domain structure, a repulsive interaction at a small $\mathrm{DB}-\mathrm{DB}$ distance $d$ is expected to favor phases with uniform coverage of DB. Therefore, we next investigate what happens if a Ge adatom is introduced in addition to an existing DB. Our calculations show that rather than bonding to $\mathrm{D}_{1}$ or $\mathrm{D}_{2} \mathrm{DB}$ atoms and forming a short $\mathrm{Ge}-$ $\mathrm{Ge}$ chain on top of them, the $\mathrm{Ge}$ adatom migrates on the germanene substrate and forms another DB. Hence, each Ge adatom introduced to the surface of germanene favors the construction of a new $\mathrm{DB}$, as long as a proper position is available. The $\mathrm{DB}-\mathrm{DB}$ coupling is calculated by placing two DBs in an $(8 \times 8)$ supercell one at a fixed corner and the 
second one placed at different sites on the supercell, as described in Figure 3. For each lateral position of the second

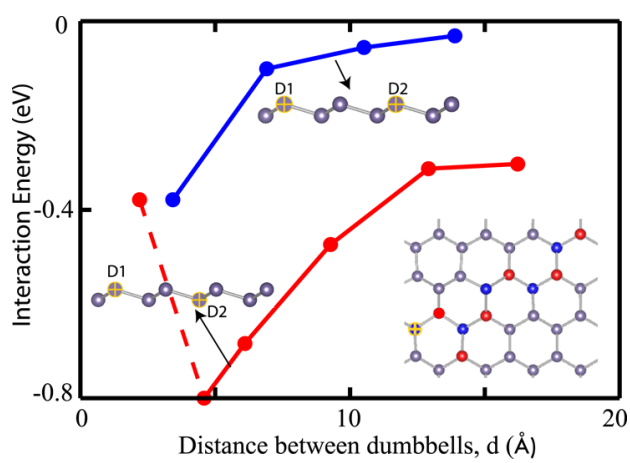

Figure 3. Interaction energy versus the distance between two DBs, $d$ on the $(8 \times 8)$ supercell of germanene. The blue and red curves represent the variation of interaction energies for DBs formed on sites with the same and opposite bucklings, respectively. The interaction energy between two DBs situated at the same buckling is set to zero for large $d$. Negative energy indicates an attractive interaction. One DB is permanently present on the yellow site, and the second $\mathrm{DB}$ is placed on various positions shown by the blue and red marks in the inset. The attractive interaction energy falls suddenly when the second DB following the red path is situated at the nearest-neighbor distance to the first DB.

$\mathrm{DB}$, the coordinates of the rest of the atoms including the height of the second DB are fully optimized. Apparently, an attractive interaction is set even for the large $\mathrm{DB}-\mathrm{DB}$ distance. The DBs tend to be close to each other and hence to form domains. However, as long as germanene continues to be fed by $\mathrm{Ge}$ adatoms, domains join to form full coverage. Here, one distinguishes two classes of sites for the second DB; one class of sites is the high buckled sites of germanene, and the other class is the low buckled sites. It should be noted that low and high buckled sites are equivalent if there is only one DB due to the upside and downside symmetry. The site-specific variation of the total energy is shown in Figure 3, where the interaction energy versus $d$ curve follows different paths. Accordingly, the formation of two DBs on sites with opposite bucklings appears to be more favorable energetically, by $\sim 0.2-0.3 \mathrm{eV}$, because the gain of energy through the buckling of bare germanene is preserved. However, this difference of energy diminishes as $d$ $\rightarrow \infty$. Notably, it is not possible to create two DBs on the nearest-neighbor sites of the lattice. This situation results in the constrained structure optimization in Figure 3 (shown by the dashed line) as a sudden fall of the attractive interaction energy when the Ge adatom is placed at the first nearest neighbor of the existing DB. The second DB tends to move to the second nearest-neighbor position when the constraints are lifted. If the second $\mathrm{DB}$ is situated at the second nearest-neighbor distance from the first DB (where both DBs are situated on the sites of the same buckling), Ge atoms of germanene at the first nearestneighbor distance to these two DBs become five-foldcoordinated.

In the case of uniform and periodic coverage, DBs form a mesh or periodically repeating supercells on germanene. The properties of the resulting germanene + DB phases depend on the size and geometry of supercells constructed from $(n \times n)$ hexagonal primitive unit cells or $(m \times n)$ rectangular unit cells of germanene, as well as the number of DBs in each supercell or the $\mathrm{DB}-\mathrm{DB}$ distance, $d$. In Table 1 , the energetics and the relevant data of the selected phases having $2 \mathrm{D}$ hexagonal or rectangular lattice structures are presented. The cohesive energy $\left(E_{\mathrm{C}}=E_{\mathrm{T}}[\mathrm{Ge}]-E_{\mathrm{T}}[\right.$ germanene $+\mathrm{DB}] / N$, ${ }^{26}$ which is the difference between the energy of one free $\mathrm{Ge}$ atom and the energy of the germanene $+\mathrm{DB}$ phase per atom or simply the energy gained per atom by constructing a particular germanene $+\mathrm{DB}$ phase) and the cohesive energy per unit area (i.e., $E_{\mathrm{C}}^{\mathrm{s}}=$ $\left.N E_{\mathrm{C}} / A\right)$ are relevant measures for energetics of the phases. In particular, $E_{C}^{s}$ is a prime criterion that decides if the phase that will grow on bare germanene. Here, $N$ is the total number of Ge atoms in the supercell of a given phase, and $A$ is the area of the supercell. Because each DB constructed on germanene lowers the energy, the FDP (full dumbbell phase), which corresponds to full coverage, attains the highest $E_{\mathrm{C}}, E_{\mathrm{C}}^{\mathrm{s}}$, and $\Delta E_{\mathrm{C}}$ among other phases listed in Table 1 . However, one Ge atom in each cell of the FDP is forced to be six-foldcoordinated, and hence, the FDP is prone to structural

Table 1. Calculated Values for the Various Phases of Germanene + DB, where DBs Form Periodically Repeating Supercells on Germanene with a 2D Hexagonal or Rectangular Lattice ${ }^{a}$

\begin{tabular}{|c|c|c|c|c|c|c|c|c|c|c|c|}
\hline lattice & mesh & $N$ & $d(\AA)$ & $A\left(\AA^{2}\right)$ & $\mu\left(\mu_{\mathrm{B}}\right)$ & $\mathrm{ES}_{\mathrm{GGA}}(\mathrm{eV})$ & $\mathrm{ES}_{\mathrm{HSE}}(\mathrm{eV})$ & $E_{\mathrm{b}}(\mathrm{eV})$ & $E_{\mathrm{C}}(\mathrm{eV})$ & $E_{\mathrm{C}}^{\mathrm{s}}$ & $\Delta E_{\mathrm{C}}(\mathrm{eV})$ \\
\hline H/FDP & $1 \times 1$ & 3 & 3.77 & 12.34 & 0 & M & M & 4.00 & 3.60 & 0.873 & 0.204 \\
\hline $\mathrm{R} / \mathrm{RDP}$ & $1 \times 1$ & 5 & 3.39 & 24.21 & 0 & M & $\mathrm{M}$ & 3.73 & 3.46 & 0.715 & 0.069 \\
\hline $\mathrm{H} / \mathrm{TDP}$ & $\sqrt{3} \times \sqrt{3}$ & 7 & 6.84 & 40.57 & 0 & M & M & 3.75 & 3.44 & 0.595 & 0.052 \\
\hline $\mathrm{H} / \mathrm{HDP}$ & $\sqrt{3} \times \sqrt{3}$ & 8 & 3.85 & 38.60 & 0 & 0.53 & 0.73 & 4.03 & 3.55 & 0.736 & 0.160 \\
\hline $\mathrm{H}$ & $2 \times 2$ & 9 & 7.79 & 52.54 & 0 & 0.16 & 0.32 & 3.72 & 3.43 & 0.585 & 0.037 \\
\hline H/DHP & $2 \times 2$ & 10 & 4.50 & 52.54 & 0 & M & 0.46 & 4.39 & 3.59 & 0.680 & 0.198 \\
\hline $\mathrm{H}$ & $3 \times 3$ & 19 & 11.68 & 118.22 & 0 & 0.29 & 0.63 & 3.54 & 3.40 & 0.551 & 0.008 \\
\hline $\mathrm{H}$ & $4 \times 4$ & 33 & 16.06 & 223.36 & 2 & $0.42 / 0.06$ & $0.77 / 0.44$ & 3.44 & 3.39 & 0.495 & 0.002 \\
\hline $\mathrm{R}$ & $2 \times 1$ & 9 & 6.95 & 48.43 & 0 & M & M & 3.85 & 3.48 & 0.189 & 0.189 \\
\hline $\mathrm{H}$ & $5 \times 5$ & 51 & 19.87 & 342.05 & 2 & $0.33 / 0.03$ & $0.61 / 0.36$ & 3.40 & 3.39 & 0.510 & 0.000 \\
\hline
\end{tabular}

${ }^{a}$ 2D Lattice: $\mathrm{H}$ hexagonal or $\mathrm{R}$ rectangular; mesh: $(m \times n)$ cell in terms of the primitive hexagonal or rectangular unit cell of germanene; $N:$ number of Ge atoms (including DB) in each supercell; $d$ : shortest distance between two DBs; $A$ : the area of the supercell; $\mu$ : magnetic moment per supercell; ES: electronic structure specified as metal $\mathrm{M}$ or a semiconductor with the band gap between the valence and conduction bands calculated by GGA and HSE (for the spin-polarized cases, the gaps between spin-up-spin-up and spin-up-spi- down bands are shown); $E_{\mathrm{b}}$ : binding energy per Ge adatom relative to germanene or the average binding energy if there are two DBs in each cell; $E_{\mathrm{C}}$ : cohesive energy (per atom) of Ge atom in the germanene $+\mathrm{DB}$ phase; $E_{\mathrm{C}}^{s}$ : cohesive energy per area; $\Delta E_{\mathrm{C}}$ : difference between the cohesive energies of a Ge atom in germanene $+\mathrm{DB}$ and in pristine germanene, where positive values indicate that the germanene $+\mathrm{DB}$ phase is favorable. For bare germanene, $E_{\mathrm{C}}=3.39 \mathrm{eV} / \mathrm{atom}$. TDP, HDP, RDP, and DHP are described in the text as well as in the Supporting Information. 
(a)

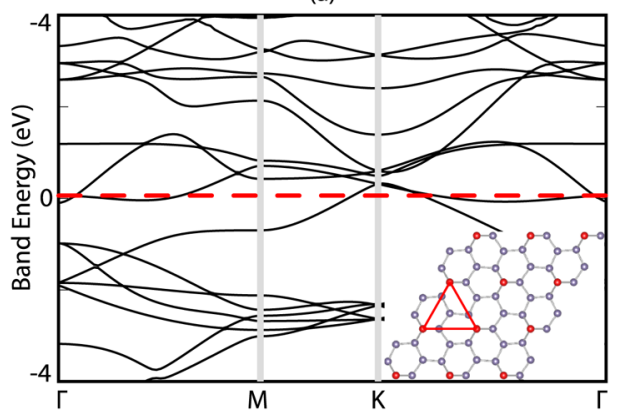

(c)

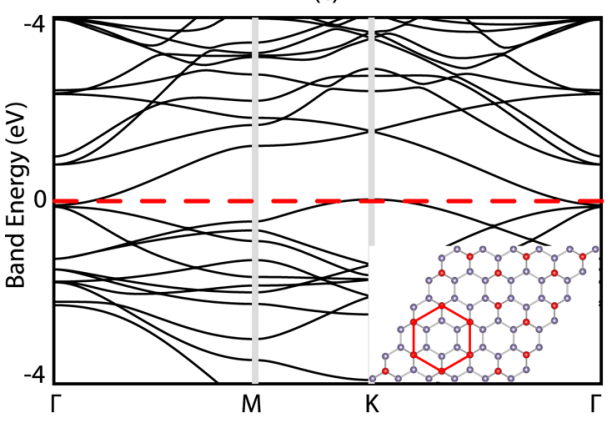

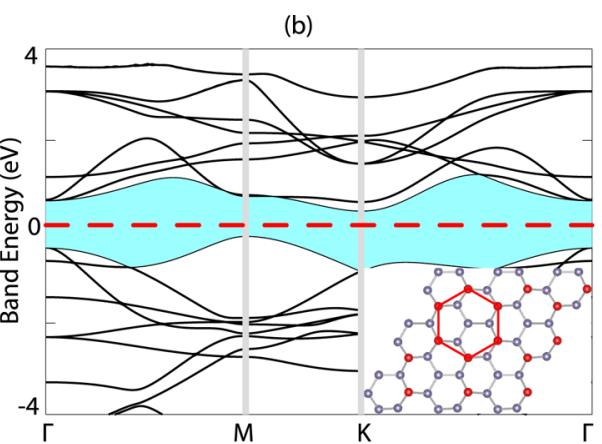

(d)

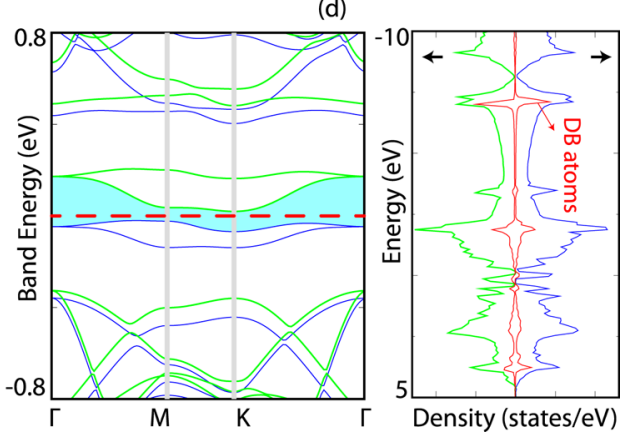

Figure 4. Electronic band structures of different phases of germanene. (a) TDP. (b) HDP. (c) DHP. (d) The triangular structure with DBs forming hexagonal $(4 \times 4)$ supercells, where the total density of states is also shown. The spin-up and spin-down bands are shown by blue and green lines, respectively. The density of states projected onto the DB atoms are shown in red and are augmented three times for a better view. Atomic structures of TDP, HDP, and DHP are described in the text as well as in the Supporting Information.

instability. In fact, our ab initio phonon calculations of this phase have branches with imaginary frequencies, which indicate structural instability, as shown in the Supporting Information. Another structure, the RDP (rectangular dumbbell phase) in Table 1 is also found to be unstable based on ab initio phonon calculations. The DHP (double hexagonal phase), where the hexagons of germanene are nested by large DB hexagons, has the highest $E_{\mathrm{b}}$ and $E_{\mathrm{C}}$ among the other stable phases listed in Table 1. Additionally, two other phases, the TDP (trigonal dumbbell phase) and HDP (hexagonal dumbbell phase), which have $(\sqrt{3} \times \sqrt{3})$ unitcells, are of particular interest because silicene grown on a $\operatorname{Ag}(111)$ substrate also showed a $(\sqrt{3} \times$ $\sqrt{3}$ ) pattern. ${ }^{16,31}$ HDP has two DBs per cell, such that DBs are situated at the corners of hexagons to form a honeycomb pattern. It appears that the HDP having the maximum cohesive energy per area, $E_{\mathrm{C}}^{s}=0.735 \mathrm{eV} / \AA^{2}$, among the other stable phases listed in Table 1 is energetically the most favorable structure to grow on bare germanene.

The germanene $+\mathrm{DB}$ phases acquire permanent magnetic moments when $d>\sim 15 \AA$, where the DB-DB coupling recedes and the $\mathrm{DB}$ behaves as a local defect on the germanene substrate with a total magnetic moment of $2 \mu_{\mathrm{B}}$ per cell. We performed additional tests to understand the magnetic order of the system. To this extent, four DB structures were created on an $(8 \times 8)$ supercell, and the ferromagnetic, antiferromagnetic, and paramagnetic states were investigated. For the ferromagnetic case, all DB atoms were given an initial spin in the same direction; for the antiferromagnetic case, opposite spins were assigned to the adjacent DBs. Our results showed that the ferromagnetic ordering has the lowest total energy, indicating that it is the most favorable configuration.

The energy difference between the ferromagnetic state and the antiferromagnetic state is $0.63 \mathrm{eV}$ per supercell. Notably, upon the relaxation of magnetic states, the final magnetic state of the structure is found to be always ferromagnetic no matter what the initial direction of spins was. The energetics of various germanene $+\mathrm{DB}$ phases display interesting trends. In general, the cohesive energy increases with increasing DB coverage, which confirms the situation in Figure 3. The binding energies, $E_{\mathrm{b}}$, also show the same trend except for DHP. The energy values presented in Table 1 imply that the higher the DB coverage of a phase, the higher its total energy gets.

DBs also modify the physical properties of germanene. In particular, the electronic and magnetic properties of pristine germanene show dramatic changes depending on the coverage (see also Table 1). First, the SOC gives rise to significant splitting in the bands of germanene-DB phases. For example, the degenerate bands at the top of the valence band of $(4 \times 4)$ mesh split by $12 \mathrm{meV}$. Normally, germanene $+\mathrm{DB}$ phases maintain their metallic state at high coverage, but they transform into a semiconductor as the size of their cell $(n)$ or the DB-DB distance $(d)$ increases, with the exception of HDP. In Figure 4, we present the energy band structures of four different germanene $+\mathrm{DB}$ phases having a hexagonal lattice. These are (i) TDP, (ii) HDP, (iii) DHP, and (iv) the triangular structure, where DBs form a $(4 \times 4)$ mesh on germanene. The band structures calculated using hybrid functionals (HSE06) are also presented as Supporting Information. It should be noted that the band gaps of the semiconductor phases are almost doubled after the HSE corrections. The dramatic effects of DB coverage is clearly seen in these band structures. While TDP is a nonmagnetic metal, HDP is a nonmagnetic semiconductor with an indirect band gap of $0.53 \mathrm{eV}$. DHP, which is a metal according to GGA, becomes a narrow band gap semiconductor after HSE06 correction. The $(4 \times 4)$ mesh of $\mathrm{DBs}$ is a magnetic, narrow band gap semiconductor. DB gives rise to localized states at $\sim-2$ and $-7 \mathrm{eV}$ below the Fermi level. 
Incidently, further to $\mathrm{Ge} \mathrm{DB}$ on germanene, $\mathrm{Si}$ adatoms on germanene can also construct asymmetric DB structures, such that $\mathrm{D}_{1}=\mathrm{Si}$ but $\mathrm{D}_{2}=\mathrm{Ge}$. The formation of this asymmetric $\mathrm{DB}$ is also exothermic and occurs spontaneously as long as a $\mathrm{Si}$ adatom on germanene is available. These asymmetric DBs as well as their periodic structures on germanene exhibit properties different from host $\mathrm{DBs}$ and multiply the functionality of the DB based on new phases of germanene. For example, the $\mathrm{Si}-\mathrm{Ge}$ asymmetric DB structure having a hexagonal lattice over the $(4 \times 4)$ supercell of germanene is a nonmagnetic metal with a binding energy of $E_{\mathrm{b}}=3.86 \mathrm{eV}$ per $\mathrm{DB}$. In contrast, the symmetric germanene-DB having the same lattice is a magnetic semiconductor, and $E_{\mathrm{b}}=3.44 \mathrm{eV}$, as shown in Table 1 . We note that like the $\mathrm{Si}-\mathrm{Ge}$ asymmetric dimer on germanene, also the $\mathrm{Ge}-\mathrm{Si}$ asymmetric dimer can be constructed on silicene spontaneously.

In conclusion, we showed that a Ge adatom constructs a $\mathrm{DB}$ structure on germanene through an exothermic and spontaneous process. Moreover, through periodically repeating decoration of DBs, new phases can grow on germanene. These stable DB-based phases exhibit diverse electronic and magnetic structures, which show remarkable changes with the coverage of DBs. In particular, the HDP, which has two DBs in each $(\sqrt{3} \times \sqrt{3})$ periodic supercell of germanene with a nearest-neighbor distance of $3.85 \AA$, attains the highest cohesive energy per unit area and forms a relatively larger honeycomb pattern over the buckled honeycomb pattern of germanene. It is an indirect band gap semiconductor and appears to be favorable energetically as compared to other phases. The DB based phases of germanene and their multilayer structures bring about a new class of single-layer materials and can be precursors of 3D layered Ge.

\section{ASSOCIATED CONTENT}

\section{S Supporting Information}

The electronic band structure of the different phases of germanene calculated with hybrid functionals, atomic structures of the various phases of germanene $+\mathrm{DB}$, and phonon dispersion curves for $\mathrm{H} / \mathrm{FDP}$ and $\mathrm{R} / \mathrm{RDP}$ structures. This material is available free of charge via the Internet at http:// pubs.acs.org.

\section{AUTHOR INFORMATION}

\section{Corresponding Author}

*E-mail: ciraci@fen.bilkent.edu.tr.

\section{Notes}

The authors declare no competing financial interest.

\section{ACKNOWLEDGMENTS}

The computational resources have been provided by TUBITAK ULAKBIM, High Performance and Grid Computing Center (TR-Grid e-Infrastructure). S.C. and V.O.O. acknowledge financial support from the Academy of Sciences of Turkey (TUBA). The authors acknowledge helpful discussions with Dr. Seymur Cahangirov, who pointed out the DHP.

\section{REFERENCES}

(1) Geim, A. K.; Novoselov, K. S. The Rise of Graphene. Nat. Mater. 2007, 6, 183.

(2) Novoselov, K. S.; Jiang, D.; Booth, T. J.; Khotkevich, V. V.; Morozov, S. V.; Geim, A. K. Two-Dimensional Atomic Crystals. Proc. Natl. Acad. Sci. U.S.A. 2005, 102, 10451.
(3) Joensen, P.; Frindt, R. F.; Morrison, S. R. Single-Layer $\mathrm{MoS}_{2}$ Mater. Res. Bull. 1986, 21, 457-461.

(4) Coleman, J. N.; Lotya, M.; O’Neill, A.; Bergin, S. D.; King, P. J.; Khan, U.; Young, K.; Gaucher, A.; De, S.; Smith, R. J.; et al. TwoDimensional Nanosheets Produced by Liquid Exfoliation of Layered Materials. Science 2011, 331, 568.

(5) Wang, Q. H.; Kalantar-Zadeh, K.; Kis, A.; Coleman, J. N.; Strano, M. S. Electronics and Optoelectronics of Two-Dimensional Transition Metal Dichalcogenides. Nat. Nanotechnol. 2012, 7, 699-712.

(6) Geim, A. K.; Grigorieva, V. Van der Waals Heterostructures. Nature 2013, 499, 419-425.

(7) Nicolosi, V.; Chhowalla, M.; Kanatzidis, M.; Strano, M. S.; Coleman, J. N. Liquid Exfoliation of Layered Materials. Science 2013, $340,6139$.

(8) Takeda, K.; Shiraishi, K. Theoretical Possibility of Stage Corrugation in Si and Ge Analogues of Graphite. Phys. Rev. B 1994, 50, 14916.

(9) Durgun, E.; Tongay, S.; Ciraci, S. Silicon and III-V Compound Nanotubes: Structural and Electronic Properties. Phys. Rev. B 2005, 72, 075420.

(10) Cahangirov, S.; Topsakal, M.; Akturk, E.; Sahin, H.; Ciraci, S. First-Principles Study of Defects and Adatoms in Silicon Carbide Honeycomb Structures. Phys. Rev. Lett. 2009, 102, 236804.

(11) Sahin, H.; Cahangirov, S.; Topsakal, M.; Bekarog Ÿlu, E.; Akturk, E.; Senger, R. T.; Ciraci, S. Monolayer Honeycomb Structures of Group-IV Elements and III-V Binary Compounds: First-Principles Calculations. Phys. Rev. B 2009, 80, 155453.

(12) Ataca, C.; Akturk, E.; Sahin, H.; Ciraci, S. Adsorption of Carbon Adatoms to Graphene and Its Nanoribbons. J. Appl. Phys. 2011, 109, 013704.

(13) Cahangirov, S.; Topsakal, M.; Ciraci, S. Armchair Nanoribbons of Silicon and Germanium Honeycomb Structures. Phys. Rev. B 2010, $81,195120$.

(14) Vogt, P.; De Padova, P.; Quaresima, C.; Avila, J.; Frantzeskakis, E.; Asensio, M. C.; Resta, A.; Ealet, B.; Le Lay, G. Silicene: Compelling Experimental Evidence for Graphenelike Two-Dimensional Silicon. Phys. Rev. Lett. 2012, 108, 155501.

(15) De Padova, P.; Quaresima, C.; Ottaviani, C.; Sheverdyaeva, P. M.; Moras, P.; Carbone, C.; Topwal, D.; Olivieri, B.; Kara, A.; Oughaddou, H.; et al. Evidence of Graphene-Like Electronic Signature in Silicene Nanoribbons. Appl. Phys. Lett. 2010, 96, 261905.

(16) Padova, P.; et al. Evidence of Dirac fermions in Multilayer Silicene. Appl. Phys. Lett. 2013, 102, 163106.

(17) Li, L.; Lu, S.; Pan, J.; Qin, Z.; Wang, Y.; Wang, Y.; Cao, G.; Du, S.; Gao, H. Buckled Germanene Formation on $\mathrm{Pt}(111)$. Adv. Mater. 2014, DOI: 10.1002/adma.201400909.

(18) Bulletin of the American Physical Society. http://meetings.aps. org/link/BAPS.2014.MAR.T51.7 (2014).

(19) Bianco, E.; Butter, S.; Jiang, S.; Restrepo, O. D.; Windi, W.; Goldberg, J. E. Stability and Exfoliation of Germanane: A Germanium Graphene Analogue. ACS Nano 2013, 7, 4414-4421.

(20) Grimme, S. J. Semiempirical GGA-Type Density Functional Constructed with a Long-Range Dispersion Correction. Comput. Chem. 2006, 27, 1787.

(21) Blochl, P. E. Projector Augmented-Wave Method. Phys. Rev. B 1994, 50, 17953.

(22) Perdew, J. P.; Burke, K.; Ernzerhof, M. Generalized Gradient Approximation Made Simple. Phys. Rev. Lett. 1996, 77, 3865-3868.

(23) Kresse, G.; Furthmuller, J. Efficient Iterative Schemes for AbInitio Total Energy Calculations Using a Plane-Wave Basis Set. Phys. Rev. B 1996, 54, 11169-11186.

(24) Heyd, J.; Scuseria, G. E.; Ernzerhof, M. Hybrid Functionals Based on a Screened Coulomb Potential. J. Chem. Phys. 2003, 118, $8207-8215$

(25) Alfe, D. PHON: A Program to Calculate Phonons Using the Small Displacement Method. Comput. Phys. Commun. 2009, 180, 2622-2633.

(26) Kittel, C. Introduction to Solid State Physics; John Wiley and Sons: New York, 1996. 
(27) Liu, C.; Jiang, H.; Yao, Y. Low-Energy Effective Hamiltonian Involving Spin-Orbit Coupling in Silicene and Two-Dimensional Germanium and Tin. Phys. Rev. B 2011, 84, 195430.

(28) Özçelik, V. O.; Gurel, H. H.; Ciraci, S. Self-Healing of Vacancy Defects in Single-Layer Graphene and Silicene. Phys. Rev. B 2013, 88, 045440.

(29) Kaltsas, D.; Tsetseris, L. Stability and Electronic Properties of Ultrathin Films of Silicon and Germanium. Phys. Chem. Chem. Phys. 2013, 15, 9710-9715.

(30) Özçelik, V. O.; Ciraci, S. Local Reconstructions of Silicene Induced by Adatoms. J. Phys. Chem. C 2013, 49, 26305-26315.

(31) Cahangirov, S.; Audiffred, M.; Tang, P.; Iacomino, A.; Duan, W.; Merino, G.; Rubio, A. Electronic Structure of Silicene on $\operatorname{Ag}(111)$ : Strong Hybridization Effects. Phys. Rev. B 2013, 88, 035432. 